\title{
Puerarin inhibits caspase-3 expression in osteoblasts of diabetic rats
}

\author{
JUNBO LIANG, HAIXIAO CHEN, WEIBO PAN and CHUNLI XU \\ Department of Orthopedics, Taizhou Hospital of Zhejiang Province, Taizhou, Zhejiang 317000, P.R. China
}

Received January 1, 2012; Accepted March 20, 2012

DOI: $10.3892 / \mathrm{mmr} .2012 .854$

\begin{abstract}
The aim of this study was to investigate the protective effect of puerarin on attenuating caspase-3 expression in osteoblasts of streptozotocin-induced diabetic rats and the possible mechanisms involved. Diabetic rats were treated with a puerarin injection of $100 \mathrm{mg} / \mathrm{kg} /$ day. After 6 weeks, the bone mineral density, pathological changes and expression of caspase- 3 were observed. The bone mineral density in the diabetic rats was significantly lower compared to the control group, while osteoblast numbers in the cortical bone were significantly reduced, and new bone formation in the diabetic rats was rare. Caspase-3 expression in the osteoblasts of diabetic rats increased compared to the rats in the control group. These pathological changes were improved and caspase- 3 expression decreased in the puerarin-treated rats compared to the diabetic rats $(\mathrm{P}<0.01)$. High glucose levels affect bone metabolism and increase caspase-3 expression in osteoblasts, and thus lead to diabetic osteoporosis. Puerarin may play a protective role in diabetic osteoporosis via the reduction of caspase-3 expression.
\end{abstract}

\section{Introduction}

An increasing number of diabetic patients are affected by chronic complications, such as cardiovascular disease, kidney disease, diabetes erectile dysfunction and diabetic osteoporosis (1-4). Osteopenia and osteoporosis are frequent complications of diabetes in both children and adults $(5,6)$. The association between diabetes and decreased bone mineral density (BMD) has been confirmed in adults, and BMD appears to be decreased in both the spine and hip in diabetic patients $(7,8)$. Diabetic populations uniformly appear to have a higher risk of hip and upper extremity fractures $(9,10)$. Evidence has indicated that apoptosis represents the most common fate of osteoblasts during physiological bone remodeling (11-13).

Correspondence to: Dr Haixiao Chen, Department of Orthopedics, Taizhou Hospital of Zhejiang Province, 150 Ximen Street, Taizhou, Zhejiang 317000, P.R. China

E-mail: liangjunbo1973@163.com

Key words: puerarin, diabetic osteoporosis, caspase-3, bone mineral density
The activation of caspase- 3 is the point of convergence of the private pathways, leading to the common executive phase of apoptosis, and ultimately cell death and BMD loss in vivo (14).

Puerarin (daidzein-8-C-glucoside) is the main isoflavone isolated from the Chinese medicinal herb, Ge-gen, the root of the wild leguminous creeper Pueraria lobata. It has been shown to be effective in the treatment of spinal ischemiareperfusion injury by reducing apoptosis (15), and protects PC12 cells against MPP(+)-induced neurotoxicity through the inhibition of the JNK signaling pathways (16). The possible mechanisms by which puerarin exerts its protective effects, at least in part, are related to its ability to increase superoxide dismutase activity and decrease the lipid peroxidation levels and apoptosis $(17,18)$. A number of studies have shown that puerarin has a similar structure to genistein (19), and isoflavones from Pueraria lobata inhibit adipogenic differentiation by bone marrow stromal cells both in vitro and in vivo and prevent alcohol-induced osteonecrosis (20). Puerarin has also been shown to promote bone formation in cultured rat osteoblasts, which may be mediated by the activation of the PI3K/ Akt pathway (21). Thus, the mechanisms by which puerarin plays its protective role in diabetic osteoporosis remain unclear.

In the present study, we investigated the protective effects of puerarin in experimental diabetic osteoporosis to determine the correlation between decreasing caspase- 3 expression in osteoblasts and diabetic osteoporosis, thus revealing an essential role for the protection of puerarin on diabetic osteoporosis.

\section{Materials and methods}

Materials. Sprague-Dawley male rats were purchased from the Experimental Animal Center of Zhejiang University. glucose electrode calibrator was obtained from MediSense (QA2583-3364; Alameda, CA, USA). Streptozotocin (STZ) was from Alexis Corporation, Lausen, Switzerland. Puerarin was purchased from Limin Pharmaceutical Corporation, Jinan, Shandong, China.

Animal model induction. Thirty Sprague-Dawley male rats were divided into 3 groups $(n=10)$ at random. The rats in the diabetic and puerarin treatment groups were fasted for $10 \mathrm{~h}$ and intraperitoneally injected with $65 \mathrm{mg} / \mathrm{kg}$ of STZ to induce diabetes; the remaining 10 control rats were also fasted for $10 \mathrm{~h}$ and injected with $0.9 \%$ saline. Forty-eight hours after the injection, blood glucose was $>16 \mathrm{mmol} / \mathrm{l}$ and urine glucose 
was $>+$ indicating that diabetes was successfully induced in the rats. The rats in the puerarin treatment group were intraperitoneally injected with puerarin $(60 \mathrm{mg} / \mathrm{kg} / \mathrm{day})$ for 6 weeks, and the other 2 groups of rats were intraperitoneally injected with saline $(6 \mathrm{ml} / \mathrm{kg} /$ day $)$. All procedures were approved by the Zhejiang University Institutional Animal Care and Ethics Committee (201002215).

Bone mineral density, $H \& E$ staining and immunohistochemistry assay. Six weeks after the injection of STZ, 10 rats per group were anesthetized, their thoracic cavities were opened and perfused intracardially with normal saline. Following saline perfusion, the animals were perfused with $300-400 \mathrm{ml}$ fixative containing $4 \%$ paraformaldehyde in $0.1 \mathrm{M}$ phosphate buffer ( $\mathrm{pH}$ 7.4). After perfusion, both the side femurs of each rat were taken out. The BMD of the left femur was determined by the small animal radiophotography system (faxitron model mx20; Faxitron Bioptics, LLC, Tucson, AZ, USA). The right femur was fixed in the same fixative for $4 \mathrm{~h}$ and then placed in $30 \%$ phosphate-buffered sucrose until the tissue sank. Sections (12 $\mu \mathrm{m}$-thick) were cut on a freezing microtome through coronary planes of proximal femur for $\mathrm{H} \& \mathrm{E}$ staining and diaminobenzidine (DAB) immunohistochemical staining.

Sections were rinsed in $0.01 \mathrm{M}$ phosphate-buffered saline (PBS) and mounted onto $0.02 \%$ poly-L-lysine-coated slides. The $\mathrm{ABC}$ system was used with $\mathrm{DAB}$ as the chromagen. Briefly, tissue sections were first washed in PBS and then incubated in $1 \%$ bovine serum albumin (BSA) for $30 \mathrm{~min}$. Tissues were then incubated overnight at $4^{\circ} \mathrm{C}$ in PBS medium with caspase-3 antibody (Boster Biotech Company, Wuhan, China) plus $1 \%$ BSA. The dilution of the primary antibody was 1:100. The control sections were incubated in PBS plus 1\% BSA. The following day, the sections were incubated in a biotinylated goat-anti-mouse secondary antibody (diluted to 1:200 in PBS; Boster Biotech Company), and subsequently in an avidinhorseradish peroxidase (HRP) solution. Immunolabeling was visualized with $0.05 \%$ DAB plus $0.3 \% \mathrm{H}_{2} \mathrm{O}_{2}$ in PBS. The sections were then dehydrated through ethanol and xylene before using cover slips.

Histomorphometrical measurements were made and the immunohistochemistry results were analyzed using caspase-3positive osteoblasts in the femur per $\mathrm{mm}^{2}$ of the 3 groups of rats using a Nikon microscope (Nikon E600; Nikon Company, Japan) connected to a RGB camera, with final magnifications, $\mathrm{x} 400$.

Statistical analysis. Data represent the means \pm SD. Treatment groups were compared using one-way ANOVA. A difference between the 3 groups was considered significant at $\mathrm{P}<0.05$. All computations were performed using SPSS 15.0 software (SPSS Inc., St. Louis, MO, USA).

\section{Results}

Body weight and blood glucose in the 3 groups. Initially, the body weight and blood glucose showed no significant differences $(\mathrm{P}>0.05)$ among the 3 groups of rats before STZ injection. At the end of 6 weeks, the puerarin treatment group had a significantly higher body weight and lower blood glucose compared to the diabetic group. Both groups also had lower
A

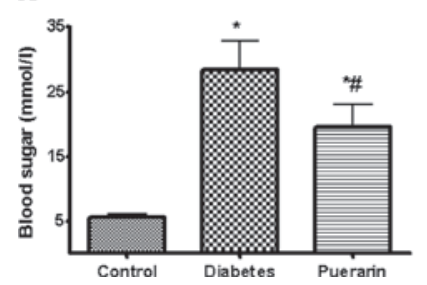

B

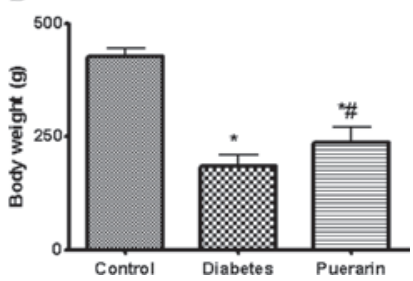

Figure 1. Body weight and blood glucose in the 3 groups 4 weeks after streptozotocin injection. (A) Blood glucose in the 3 groups; (B) Body weight of the 3 groups.

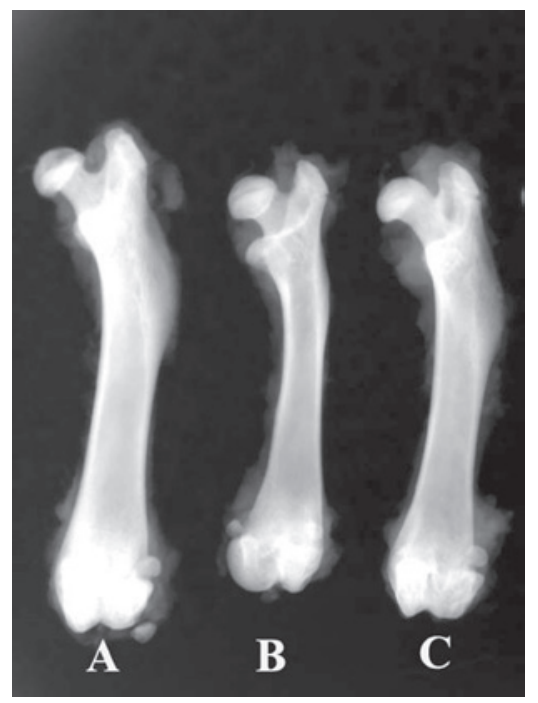

Figure 2. X-ray images of the left femur isolated from the 3 groups. (A) Control rat. (B) Diabetic rat showed more bone loss and the length was reduced. (C) Puerarin-treated rat showed less bone loss and the length was prolonged compared to the diabetic rat.

body weight and higher blood glucose than the control group $(\mathrm{P}<0.01)$ (Fig. 1).

Proximal femoral BMD analysis. Compared to the nondiabetic control rats, the STZ-treated rats showed a decrease in femoral BMD $(-15.4 \pm 2.3 \%$ vs. control, $\mathrm{P}<0.05)$. By contrast, the BMD in diabetic rats treated with puerarin increased $(9.7 \pm 2.1 \%$ vs. diabetes, $\mathrm{P}<0.05)$ compared to the diabetic rats, but was still lower than that of the control rats $(-7.2 \pm 1.1 \%$ vs. control, $\mathrm{P}<0.05$ ) (Fig. 2).

$H \& E$ staining and immunohistochemistry assay. Osteoblasts were identified by a central lucency, eccentric nucleus and basophilic cytoplasmic stain. Osteoclasts were identified as single or multi-nucleated cells demonstrating a foamy cytoplasm. After 6 weeks of inducing diabetes, the proximal femur H\&E staining demonstrated that there was an increased number of osteoclasts clumped together, cortical bone reduction, and deteriorated bone micro-architecture in the diabetic rats. The overall growth plate architecture was dominated by hypertrophic chondrocytes, disrupted with fewer proliferative and chondroblastic cells as compared to the control rats. The femurs of the normal control rats had a normal shape with an intact cartilage, qualitatively equivalent cortical bone, and a 


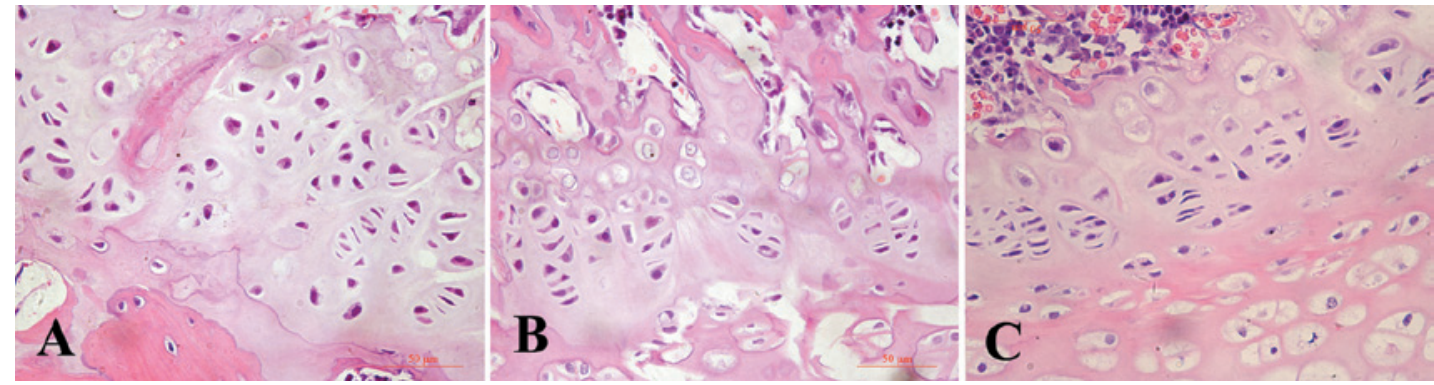

Figure 3. H\&E staining of the proximal femur tissues (magnification, x400). (A) Control rat tissue. (B) Diabetic rat tissue showing more osteoclasts that were clumped together and cortical bone reduction; the overall growth plate architecture was dominated by hypertrophic chondrocytes, with rare new bone formation and disrupted by fewer proliferative and chondroblastic cells. (C) Puerarin-treated rat tissue showing less osteoclasts that were clumped together, more proliferative and chondroblastic cells and a well-organized trabecular bone.
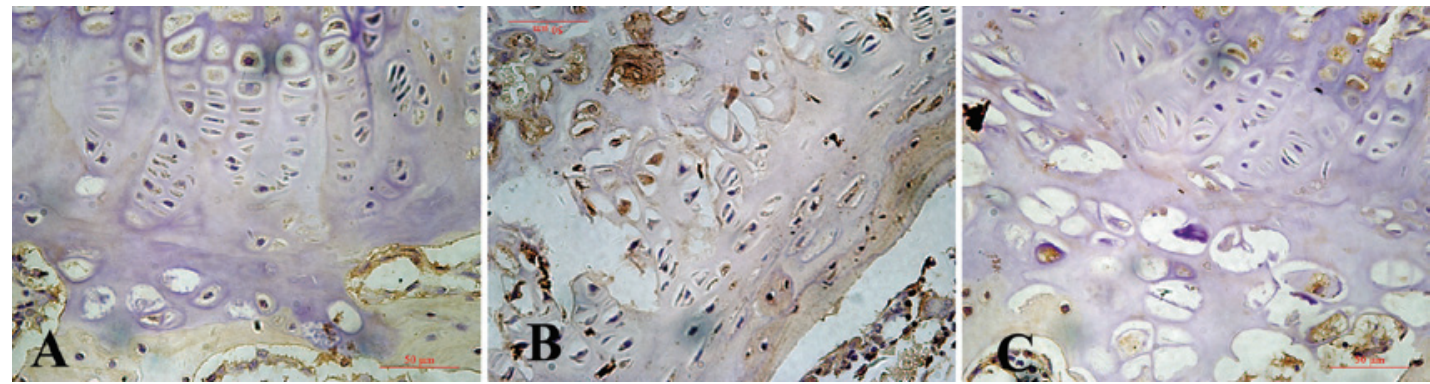

Figure 4. Expression of caspase- 3 in the proximal femur tissue of the 3 groups as shown by immunohistochemistry staining (magnification, $\mathrm{x} 400$ ). (A) Control rat showed few caspase-3-positive osteoblasts. (B) Diabetic rat tissue showing more caspase-3-positive osteoblasts in the overall growth plate. (C) Puerarin treatment reduced caspase-3-positive osteoblasts.

well-organized bone matrix composed of trabecular bone compared to the diabetic rats, and these pathological changes were less evident in the puerarin-treated group (Fig. 3).

Fig. 4 shows light photomicrographs of caspase-3 immunohistochemical staining of the osteoblasts from the 3 groups of rats. Osteoblastic cells that stained positive for caspase- 3 showed buff-coloured granules with DAB staining. Increased caspase-3 expression was found in the diabetic rats compared to the control group. The puerarin-treated animals had a significantly decreased number of caspase-3-positive osteoblasts $\left(10.9 \pm 1.2 \times 10^{3} / \mathrm{mm}^{2}\right)$, while compared to the diabetic rats $\left(16.0 \pm 1.5 \times 10^{3} / \mathrm{mm}^{2}\right)$, the caspase-3-positive osteoblasts in the control group were $7.1 \pm 0.8 \times 10^{3} / \mathrm{mm}^{2}(\mathrm{P}<0.01)$.

\section{Discussion}

Patients with diabetes are at greater risk of fractures due to a low BMD. Lower BMD is explained by insulinopenia and hyperglycemia, which impair bone formation (22). Despite discrepancies between BMD and fracture rates, clinical trials uniformly support the fact that new bone formation, bone micro-architecture and bone quality are altered in both types of diabetes (type 1 and 2) $(23,24)$. Modern pharmacology has demonstrated that puerarin activates blood and eliminates stasis, improves micro-circulation and decreases blood glucose $(25,26)$. Furthermore, it has been shown to have clinical applications in the treatment and management of diabetes, cardiovascular diseases and osteoporosis of post-menopausal women (27-29). In the present study, STZ-treated rats showed a decrease in femoral BMD compared to normal control rats. By contrast, BMD in diabetic rats treated with puerarin increased when compared to the diabetic rats. More osteoblasts and less osteoclasts were found in the puerarin-treated group than in diabetic rats. The femurs of the diabetic rats showed reduced cortical bone, deteriorated bone micro-architecture and decreased epiphyseal and metaphyseal trabecular width, compared to the normal rats; thus this damage was prevented in the puerarin-treated group.

Activation of caspase- 3 is the point of convergence of the private pathways, after which specific substrates are cleaved leading to the common executive phase of apoptosis. The end-result of this cascade is chromatin condensation, nuclear fragmentation, increase in cell membrane permeability and ultimately cell death, and apoptosis of caspase- 3 is the main executive element (30). Previous studies have found that caspase- 3 is crucial for the differentiation of bone marrow stromal stem cells by influencing the TGF- $\beta / \operatorname{Smad} 2$ pathway and cell cycle progression (31). In glucocorticoid-induced osteoporosis, caspase-3 activation has been shown to lead to mouse osteoblastic cell apoptosis (32). In the diabetic osteoporosis rat model, we found that the expression of caspase- 3 in the osteoblasts was significantly higher in the diabetic rats than in normal rats. Therefore, we speculate that high blood sugar diabetes status contributes to the high expression of caspase-3, while the high expression of caspase-3 promotes the apoptosis of osteoblasts that destroyed the couples of osteoblasts and osteoclasts, leading to the occurrence of osteoporosis. However, puerarin treatment decreased caspase-3 expression in osteoblasts, inhibited apoptosis and prevented the damages in bone metabolism. 
In summary, the present study shows that puerarin treatment results in the improvement of the subchondral area of bone cell shrinkage, osteoblast growth, more proliferative and chondroblastic cells and well-organized trabecular bone by inhibiting caspase-3 expression. However, further studies are required to confirm which signaling pathway is involved in the puerarin inhibition of caspase- 3 expression in osteoblasts under hyperglycemia.

\section{References}

1. Zhang XM, Shi PH, Cao SH, Yu HJ, Azad J and Ling SC: Expression changes of transforming growth factor-betal and thrombospondin-1 in cavernous tissues of diabetic rats. Urol Int 84: 221-225, 2010.

2. Del Canizo Gomez FJ, Fernandez Perez C, Moreno Ruiz I, et al: Microvascular complications and risk factors in patients with type 2 diabetes. Endocrinol Nutr 58: 163-168, 2011.

3. Lu B, Gong W, Yang Z, et al: An evaluation of the diabetic kidney disease definition in chinese patients diagnosed with type 2 diabetes mellitus. J Int Med Res 37: 1493-1500, 2009.

4. Atsumi H, Kitada M, Kanasaki K and Koya D: Reversal of redoxdependent inhibition of diacylglycerol kinase by antioxidants in mesangial cells exposed to high glucose. Mol Med Report 4: 923-927, 2011.

5. Viegas M, Costa C, Lopes A, Griz L, Medeiro MA and Bandeira F: Prevalence of osteoporosis and vertebral fractures in postmenopausal women with type 2 diabetes mellitus and their relationship with duration of the disease and chronic complications. J Diabetes Complications 25: 216-221, 2011.

6. Hofbauer LC, Brueck CC, Singh SK and Dobnig H: Osteoporosis in patients with diabetes mellitus. J Bone Miner Res 22: 1317-1328, 2007.

7. Yamamoto M, Yamaguchi T, Yamauchi M, Kaji $H$ and Sugimoto T: Diabetic patients have an increased risk of vertebral fractures independent of BMD or diabetic complications. J Bone Miner Res 24: 702-709, 2009.

8. Miazgowski T, Pynka S, Noworyta-Zietara M, KrzyzanowskaSwiniarska B and Pikul R: Bone mineral density and hip structural analysis in type 1 diabetic men. Eur J Endocrinol 156: 123-127, 2007.

9. Norris R and Parker M: Diabetes mellitus and hip fracture: a study of 5966 cases. Injury 42: 1313-1316, 2011.

10. Yano S and Sugimoto T: Fracture risk in secondary osteoporosis. Clin Calcium 20: 1369-1377, 2010 (In Japanese).

11. Hie M, Iitsuka N, Otsuka T and Tsukamoto I: Insulin-dependent diabetes mellitus decreases osteoblastogenesis associated with the inhibition of Wnt signaling through increased expression of Sost and Dkk1 and inhibition of Akt activation. Int J Mol Med 28: 455-462, 2011.

12. Lana-Elola E, Rice R, Grigoriadis AE and Rice DP: Cell fate specification during calvarial bone and suture development. Dev Biol 311: 335-346, 2007.

13. Canalis E: The fate of circulating osteoblasts. N Engl J Med 352: 2014-2016, 2005.

14. Chan WH, Wu HJ and Shiao NH: Apoptotic signaling in methylglyoxal-treated human osteoblasts involves oxidative stress, c-Jun N-terminal kinase, caspase-3, and p21-activated kinase 2. J Cell Biochem 100: 1056-1069, 2007.
15. Tian F, Xu LH, Zhao W, Tian LJ and Ji XL: The optimal therapeutic timing and mechanism of puerarin treatment of spinal cord ischemia-reperfusion injury in rats. J Ethnopharmacol 134: 892-896, 2011.

16. Wang G, Zhou L, Zhang Y, et al: Implication of the c-Jun$\mathrm{NH}$-terminal kinase pathway in the neuroprotective effect of puerarin against 1-methyl-4-phenylpyridinium $\left(\mathrm{MPP}^{+}\right)$-induced apoptosis in PC-12 cells. Neurosci Lett 487: 88-93, 2010.

17. Chung MJ, Sung NJ, Park CS, et al: Antioxidative and hypocholesterolemic activities of water-soluble puerarin glycosides in HepG2 cells and in C57 BL/6J mice. Eur J Pharmacol 578: $159-170,2008$.

18. Xu X, Zhang S, Zhang L, Yan W and Zheng X: The Neuroprotection of puerarin against cerebral ischemia is associated with the prevention of apoptosis in rats. Planta Med 71: 585-591, 2005.

19. Hwang YP and Jeong HG: Mechanism of phytoestrogen puerarin-mediated cytoprotection following oxidative injury: estrogen receptor-dependent up-regulation of PI3K/Akt and HO-1. Toxicol Appl Pharmacol 233: 371-381, 2008.

20. Wang Y, Yin L, Li Y, Liu P and Cui Q: Preventive effects of puerarin on alcohol-induced osteonecrosis. Clin Orthop Relat Res 466: 1059-1067, 2008.

21. Zhang Y, Zeng X, Zhang L and Zheng X: Stimulatory effect of puerarin on bone formation through activation of PI3K/Akt pathway in rat calvaria osteoblasts. Planta Med 73: 341-347, 2007.

22. Follak N, Kloting I and Merk H: Influence of diabetic metabolic state on fracture healing in spontaneously diabetic rats. Diabetes Metab Res Rev 21: 288-296, 2005.

23. Abbassy MA, Watari I and Soma K: The effect of diabetes mellitus on rat mandibular bone formation and microarchitecture. Eur J Oral Sci 118: 364-369, 2010.

24. Yamaguchi T, Yamamoto M, Kanazawa I, et al: Quantitative ultrasound and vertebral fractures in patients with type 2 diabetes. J Bone Miner Metab 29: 626-632, 2011.

25. Chang Y, Hsieh CY, Peng ZA, et al: Neuroprotective mechanisms of puerarin in middle cerebral artery occlusion-induced brain infarction in rats. J Biomed Sci 16: 9, 2009.

26. Chen WC, Hayakawa S, Yamamoto T, Su HC, Liu IM and Cheng JT: Mediation of beta-endorphin by the isoflavone puerarin to lower plasma glucose in streptozotocin-induced diabetic rats. Planta Med 70: 113-116, 2004.

27. Manonai J, Chittacharoen A, Udomsubpayakul U, Theppisai H and Theppisai U: Effects and safety of Pueraria mirifica on lipid profiles and biochemical markers of bone turnover rates in healthy postmenopausal women. Menopause 15: 530-535, 2008.

28. Wong KH,Li GQ,Li KM, Razmovski-Naumovski V and Chan K: Kudzu root: traditional uses and potential medicinal benefits in diabetes and cardiovascular diseases. J Ethnopharmacol 134: 584-607, 2011.

29. Mun JG, Grannan MD, Lachcik PJ, et al: Tracking deposition of a 14C-radiolabeled kudzu hairy root-derived isoflavone-rich fraction into bone. Exp Biol Med (Maywood) 235: 1224-1235, 2010.

30. Slee EA, Adrain C and Martin SJ: Executioner caspase-3, -6, and -7 perform distinct, non-redundant roles during the demolition phase of apoptosis. J Biol Chem 276: 7320-7326, 2001.

31. Miura M, Chen XD, Allen MR, et al: A crucial role of caspase-3 in osteogenic differentiation of bone marrow stromal stem cells. J Clin Invest 114: 1704-1713, 2004

32. Chua CC, Chua BH, Chen Z, Landy C and Hamdy RC: Dexamethasone induces caspase activation in murine osteoblastic MC3T3-E1 cells. Biochim Biophys Acta 1642: 79-85, 2003. 